\title{
Uma abordagem de decisão multi-critério para preempção de caminhos de luz em EON
}

\author{
Alex S. Santos ${ }^{1}$, Gustavo B. Figueiredo ${ }^{1}$ \\ ${ }^{1}$ Instituto de Matemática e Estatística - Universidade Federal da Bahia (UFBA) \\ Av. Adhemar de Barros, s/n, Ondina - 40170-115 - Salvador - BA - Brasil \\ santos.alex@ufba.br, gustavo@dcc.ufba.br
}

\begin{abstract}
Some approaches like service degradation have been presented to minimize the blocking probability caused by insufficient network resource. In service degradation, the requests can receive bandwidth lower than was solicited. Situations where there is no option of requests to perform service degradation, is a limitation for this approach. Another way to free optical resource is to perform lightpath preemption, which has different quality of service $(Q o S)$ requirements. These QoS parameters should be taken into account when performing preemption and the problem of choosing what lightpath to be preempted becomes a multi-criteria decision making. We propose an algorithm that uses hybrid Multi-Attribute Decision Making (MADM) technique to perform lightpath preemption in EON. This technique is comprised of an Analytical Hierarchical Process (AHP) and Grey Relational Analysis (GRA). Simulations were carried out in the national science foundation network (NSFNET) topology to evaluate the proposed algorithm. The results show that the proposed approach can reduce the blocking probability of the highest priority service class.
\end{abstract}

Resumo. Redes ópticas elásticas possuem a capacidade de oferecer largura próxima da solicitada pelo cliente. O que permite tal característica é a baixa granularidade dos recursos espectrais. Na degradação de serviço, requisições podem receber largura de banda menor que a solicitada. No entanto, situação onde não há opções de caminhos de luz para realizar a degradação é uma limitação para esta abordagem. Outra abordagem que pode ser adotada para liberar recursos ópticos é a realização de preempção dos caminhos de luz, os quais possuem diferentes requerimentos de qualidade de serviço (QoS). Tais parâmetros de QoS devem ser levados em consideração quando a preempção é realizada e a escolha de qual caminho de luz deve sofrer preempção se torna um problema de tomada de decisão multi-critério. Nós propomos um algoritmo que utiliza a técnica hybrid Multi-Attribute Decision Making (MADM) para realizar preempção de caminhos de luz em EON. Esta técnica é composta pelo Analytical Hierarchical Process (AHP) e Grey Relational Analysis (GRA). Simulações foram realizada na topologia de rede national science foundation network (NSFNET) para avaliar o algoritmo proposto. Os resultados mostraram que a abordagem proposta foi capaz de reduzir a probabilidade de bloqueio das classes de serviço com maior prioridade.

\section{Introdução}

Analisando a perspectiva do operador da rede, a interrupção de um serviço é o resultado menos desejado para alguma ação tomada na rede. Desta forma, operadores de rede 
tentam evitar ao máximo ações que produzam interrupção de serviço. No entanto, quando a ocupação da rede alcança o seu limite máximo, é necessário decidir se um caminho de luz deve sofrer preempção ou realizar o bloqueio de uma nova requisição. Esta decisão é necessária porque em determinados momentos, as abordagens utilizadas para lidar com a falta de recursos da rede falham em evitar o bloqueio de novas requisições de conexões devido a falta de caminhos de luz disponíveis para realizar a degradação.

Para abordar este problema, algumas abordagens para preempção de caminhos de luz tem sido propostas na literatura [Szymanski et al. 2007, Fawaz et al. 2008]. No entanto, estas abordagens fazem sua decisão de preempção baseadas somente na prioridade dos caminhos de luz ou qualidade de serviço (do inglês Quality of Service (QoS)). No entanto, a decisão de qual caminho de luz deve sofrer preempção pode levar em consideração diversos fatores como: prioridade, tempo de finalização, número de saltos e quantidade de recursos espectrais solicitados. Por exemplo, caminhos de luz que ocupam maiores quantidades de espectro devem ser mais adequados para a preempção uma vez que eles terão a capacidade de liberar maiores quantidades de recursos ópticos. De forma semelhante temos os caminhos de luz que possuem um número maior de saltos. Pode-se dar preferência também a caminhos de luz que possuem menor tempo de finalização da conexão em detrimento à aqueles com tempo de finalização mais longo.

Este é um problema de tomada de decisão multi-critério que na maioria das vezes deve ser resolvido em tempo real pelos operadores de rede e que na maioria das vezes contam com apenas sua experiência como ferramenta de suporte. Além disso, a quantidade de parâmetros envolvidos na tomada de decisão pode variar de acordo com o cenário provocado pela falha ou o contexto do operador da rede, os quais definem a importância relativa de cada fator adotado. Desta forma, a falta de percepção do contexto dificulta a utilização de métodos quantitativos puros na solução do problema.

Neste trabalho propomos um algoritmo que utiliza os métodos Analytic Hierarchy Process (AHP) [Saaty 1990] e Grey Relational Analysis (GRA) [Q. and A. 2005] para decidir sobre a preempção de caminhos de luz em Redes Ópticas Elásticas (do inglês Elastic Optical Networks (EON)). A abordagem proposta se caracteriza por possuir baixa complexidade computacional e ser ciente do contexto, sendo assim ajustável para o uso prático dos operadores de rede. Além disso, esta abordagem pode ser adotada em vários cenários, considerando os conjunto de parâmetros com diferente importância relativa.

Para realização das simulações, nós consideramos uma rede óptica elástica operando em um cenário dinâmico, onde requisições imediatas (do inglês Imediate Requests (IR)) [Lu et al. 2015] são classificadas em três tipos de classes de serviço (do inglês Classe of Service (CoS)) e possuem tempo de duração desconhecidos. Consideramos também que requisições de alta e baixa prioridade podem realizar a preempção de caminhos de luz provisionados. Além disso, a tomada de decisão da preempção é realizada após a análise multi-critério. Os resultados apontam que a abordagem proposta é capaz de reduzir a probabilidade de bloqueio geral da rede e também das requisições de alta prioridade, levando a rede a um aumento da taxa de aceitação de requisições.

Este trabalho é organizado da seguinte forma: Na seção 2 os trabalhos relacionados são apresentados. Na seção 3 descrevemos a abordagem proposta, o AHP e o GRA. $\mathrm{Na}$ seção 4 os resultados obtidos são discutidos. Finalmente, na seção 6 o trabalho é 
concluído.

\section{Trabalhos relacionados}

Os serviços que são oferecidos na rede possuem diferentes requisitos de QoS que devem ser atendidos. Estes requerimentos podem ser descritos em um contrato de nível de serviço (do inglês Service Level Agreement (SLA)) [Fawaz et al. 2004]. Como estes serviços são heterogêneos, é necessário o emprego de mecanismos para realizar a diferenciação adequada das $\mathrm{CoS}$. A diferenciação dos caminhos de luz pode ser realizada em pelo menos três formas: preservando os recursos da rede para caminhos de luz de alta prioridade; utilizando diferentes algoritmos de roteamento ou rotas candidatas para cada classe de requisição ou realizando a preempção e rerroteamento de caminhos de luz de baixa prioridade para aceitar os de alta prioridade [Szymanski et al. 2007]. Em casos de falta de recursos, a primeira e segunda opção podem não ser suficientes para evitar o bloqueio das novas requisições que chegam à rede, restando apenas a última opção a ser adotada.

Com o objetivo de evitar cenários altamente desequilibrados causados por pares de nós que bloqueiam o estabelecimento de novos caminho da luz, os autores em [Sivasankaran et al. 2012] propuseram um mecanismo de preempção baseado em restauração que visa rerrotear caminhos de luz críticos que foram desconectados devido à falhas na rede. Os autores em [Fawaz et al. 2008] propuseram uma nova estratégia de gerenciamento de configuração de conexão com o objetivo de diferenciar a probabilidade de bloqueio de caminhos de luz pertencentes a diferentes classes de serviço. Nesta abordagem, caminhos de luz de alta prioridade recebem melhor tratamento que os de baixa prioridade. A proteção dos caminhos de baixa prioridade é realizada través de rerroteamento. Três estratégias de preempção foram propostas: soft, normal e hard.

É importante mencionar que nenhuma das estratégias mencionadas anteriormente levam em consideração os múltiplos parâmetros e suas interações, os quais podem influenciar a decisão da realização da preempção. Como diversos parâmetros devem ser levados em consideração, acreditamos que o uso conjunto do AHP-GRA possa ajudar em uma tomada de decisão mais adequada.

\section{Abordagem proposta}

Nesta seção descreveremos os conceitos fundamentais do AHP-GRA e como a tomada de decisão entre o bloqueio de uma nova requisição ou a preempção de um caminho de luz provisionado é realizada.

\subsection{Analytic Hierarchy Process}

O AHP é uma técnica de análise que arranja os fatores envolvidos no processo de tomada de decisão em uma estrutura de hierarquia descendente, organizados em níveis sucessivos, iniciando do objetivo para os critérios, subcritérios e alternativas. O objetivo é dividir um problema complicado em subproblemas menos complicados que recebem o nome de fatores de decisão. Os fatores de decisão por sua vez, são ponderados de acordo com sua dominância relativa para o problema. A Figura 1 apresenta um exemplo de uma hierarquia de decisão com três níveis, onde o objetivo é fazer o bloqueio de uma requisição ou a preempção de um caminho de luz e os critérios adotados para esta decisão são CoS, 
holding-time, largura de banda, e quantidade de saltos. Além disso, existem três alternativas: requisição $i$, caminho de luz 1 e caminho de luz 2. Para realizar a escolha das alternativas, o AHP segue 4 etapas que são: $i$ ) decomposição, $i i$ ) comparação pareada, iii) cálculo das prioridades relativas e $i v$ ) cálculo do peso de decisão para cada alternativa [Q. and A. 2005].

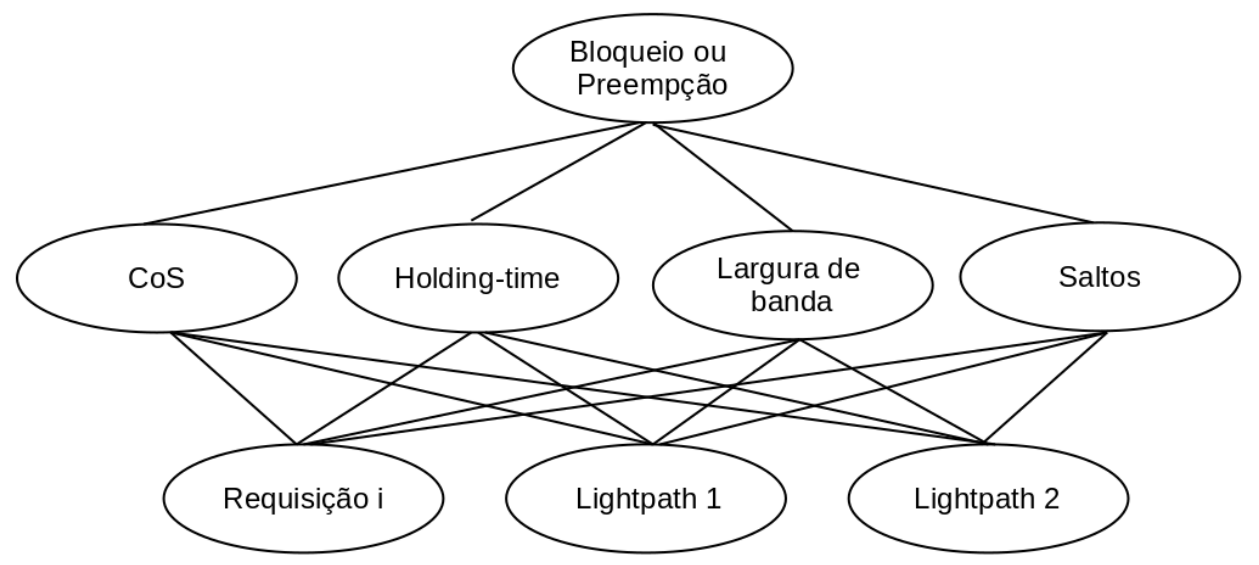

Figura 1. Hierarquia de decisão para a preempção de caminhos de luz.

$\mathrm{Na}$ fase de decomposição são identificados os fatores de decisão e então eles são estruturados em uma hierarquia de decisão. O objetivo do problema é colocado no topo da hierarquia, os fatores de decisão são colocados no nível intermediário e as alternativas de decisão ficam na base. No segundo passo é a realização da comparação pareada. Nesta etapa, cada fator é comparado com os demais fatores presentes no mesmo nível da hierarquia. Uma escala fundamental que varia de 0 a 9 é utilizada para realizar esta comparação. O objetivo desta escala é determinar o quão importante é um fator sobre o outro e de forma geral, ela pode ser expressa como: 1 - Igual importância; 3 - Importância moderada; 5 Alta importância; 7 - Muito alta importância e 9 - Extrema alta importância. Os valores 2, 4, 6 e 8 são considerados de importância intermediária [Q. and A. 2005].

Na terceira etapa é calculada a importância relativa dos fatores de decisão de cada nível da hierarquia. Para isso, é utilizada uma matriz AHP onde linhas e colunas representam os fatores de decisão. É importante lembrar que a atribuição dos níveis adequados de importância é baseada no conhecimento prévio do estruturador do problema (o operador da rede). Ao final, obtemos uma matriz recíproca onde as entradas são simétricas com respeito à diagonal principal [Q. and A. 2005, Liu et al. 2012]. Como a matriz AHP é construída através de preferências humanas, medidas para evitar erros de decisão são adotadas. Para isso, deve-se checar a consistência da matriz AHP através do índice de consistência (do inglês Consistency Index (CI)), o qual é calculado através da Equação 1

$$
C I=\frac{\left(\lambda_{\max }-n\right)}{(n-1)}
$$

onde $\lambda_{\max }$ é maior autovalor da matriz AHP e $n$ é o número de critérios sendo comparados. A matriz AHP é considerada perfeitamente consistente se CI é igual a 0 . Se o valor de CI for diferente de 0 , ele deve ser positivo e neste caso, a taxa de consistência (do inglês Consistency Ratio (CR)) deve ser calculada. O CR é calculado através da 
divisão entre CI e o índice randômico (do inglês Random Index (RI)), o qual é definido como o CI médio de uma matriz AHP gerada randomicamente (Tabela 1). Se RI $\leq 0.1$ a estimativa é considerada aceitável. Caso contrário, a comparação precisa ser ajustada. A última etapa computa o peso geral de cada alternativa de decisão através do produto dos pesos locais de cada nível [Q. and A. 2005].

Tabela 1. Índice randômico (RI) obtido da média de um elevado número de matizes recíprocas [Saaty 1990].

\begin{tabular}{|c|c|c|c|c|c|c|c|c|c|}
\hline Matriz & 1 & 2 & 3 & 4 & 5 & 6 & 7 & 8 & $\ldots$ \\
\hline RI & 0 & 0 & 0.58 & 0.9 & 1.12 & 1.24 & 1.32 & 1.41 & $\ldots$ \\
\hline
\end{tabular}

\subsection{Grey Relational Analysis}

Após o uso do AHP para encontrar o peso relativo de cada fator de decisão, o método GRA é usado para analisar a correspondência entre sequências discretas que representam cada alternativa de decisão [Q. and A. 2005]. Este método é útil para representar o quão próximas estão as sequências que representam as alternativas de decisão de uma sequência de referência que tem o melhor valor para cada critério adotado.

No método GRA são construídos níveis de similaridade entre diferentes elementos das sequências e cada elemento é comparado quantitativamente. A sequência considerada referência possui os valores ideais para cada critério. As demais sequências possuem os valores a serem comparados. O Gray Relational Coefficient (GRC) determina a relação entre as sequências comparadas e aquela com o maior GRC é a preferida. São necessários 4 passos para implementar o GRC: 1-Definir a série de referência e as séries a serem comparadas; 2-Normalizar as entidades individuais; 3-Cálculo dos GRCs e 4-Seleção da sequência com maior GRC [Liu et al. 2012].

No primeiro passo, definimos a sequência de referência e as sequências a serem comparadas. Desta forma, definimos $x_{0}$ que representa a sequência com valor ideal para cada alternativa e $x_{i}$ as sequências a serem comparadas. Além disso, cada sequência possui $k$ elementos ou entidades individuais.

$$
\begin{gathered}
x_{0}=\left(x_{0}(1), x_{0}(2), \ldots, x_{0}(k)\right) \\
x_{i}=\left(x_{i}(1), x_{i}(2), \ldots, x_{i}(k)\right)
\end{gathered}
$$

O segundo passo é a normalização dos dados. Três situações são consideradas para evitar a distorção dos dados normalizados: maior-melhor, menor-melhor e nominal-melhor. A sequência de referência $x_{0}$ contém o limite superior, inferior ou moderado quando alguma das três abordagens são utilizadas. As fórmulas utilizadas para calcular tais situações são [Q. and A. 2005]

$$
\begin{gathered}
x_{i}^{*}(j)=\frac{x_{i}(j)-l_{j}}{u_{j}-l_{j}}, \\
x_{i}^{*}(j)=\frac{u_{j}-x_{i}(j)}{u_{j}-l_{j}}, \\
x_{i}^{*}(j)=1-\frac{\left|x_{i}(j)-m_{j}\right|}{\max \left\{u_{j}-m_{j}, m_{j}-l_{j}\right\}},
\end{gathered}
$$


onde $u_{j}$ e $l_{j}$ são definidos como:

$$
\begin{gathered}
u_{j}=\max \left\{x_{1}(j), x_{2}(j), \ldots x_{n}(j)\right\}, \\
l_{j}=\min \left\{x_{1}(j), x_{2}(j), \ldots x_{n}(j)\right\},
\end{gathered}
$$

e $m_{j}$ é o valor desejado quando a situação nominal-melhor é utilizada, e $j=1,2, \ldots k$. No terceiro passo, o GRC é calculado por

$$
G R C_{i}=\frac{1}{m} \sum_{j=1}^{m} \frac{\Delta_{\min }+\Delta_{\max }}{\Delta_{i}+\Delta_{\max }}
$$

onde $\Delta_{i}=\left|x_{0}^{*}(j)-x_{i}^{*}(j)\right|, \Delta_{\max }=\max _{(i, j)}\left(\Delta_{i}\right)$ e $\Delta_{\min }=\min _{(i, j)}\left(\Delta_{i}\right)$, onde $\max _{(i, j)}$ e $\min _{(i, j)}$ são funções para calcular os valores máximo e mínimos de um conjunto de números independentes variando com $i$ e $j$. A sequência com maior GRC será a preferida.

\subsection{Preempção de caminhos de luz usando o AHP-GRA}

O AHP-GRA são importantes ferramentas que nos ajudam a decidir entre diversos fatores de decisão o qual nos dá o melhor resultado baseados na percepção da rede, Além disso, devido à subjetividade (flexibilidade) na importância de cada fator, esta decisão pode variar dependendo da necessidade do tomador de decisão. O esquema de tomada de decisão para o bloqueio ou preempção de um caminho de luz é apresentado na Figura 2. É importante salientar que quando um caminho de luz sofre preempção, é realizada uma tentativa de rerroteamento no segundo menor caminho entre seus pares de nós origemdestino. Se esta tentativa falhar, a requisição é então bloqueada.

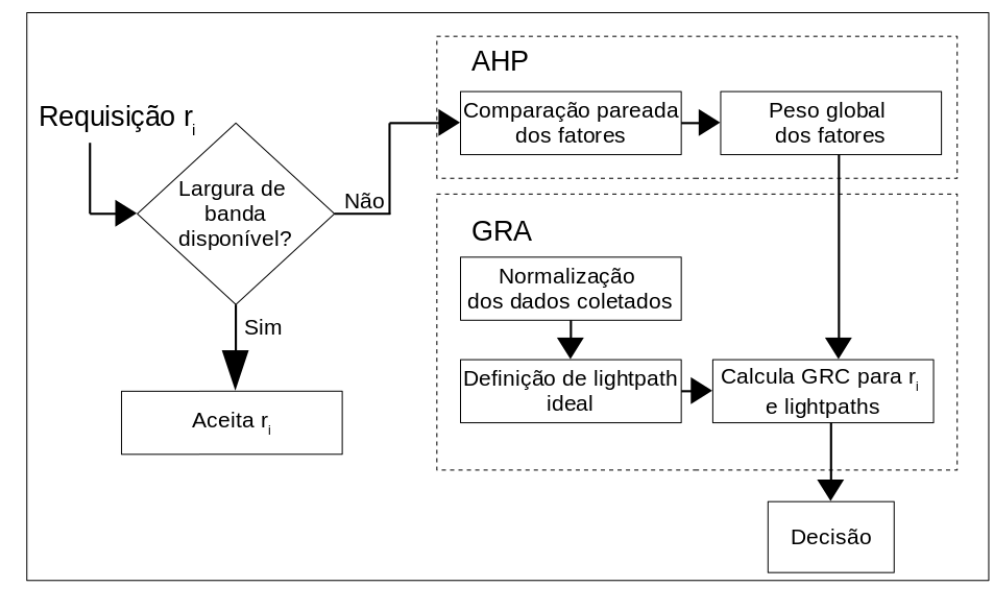

Figura 2. Esquema de tomada de decisão para preempção de caminhos de luz.

A Tabela 2 apresenta a comparação pareada para os critérios adotados na escolha entre bloquear uma nova requisição ou realizar a preempção de um caminho de luz provisionado. É também apresentado o peso de cada fator. Como $C I=0,0642 \mathrm{e}$ $C R=0,0714$, concluímos que a matriz AHP é consistente. O peso final de cada critério (ou vetor de prioridade) é dado por: $\mathbf{w}_{\mathbf{p}}=\{0.1504,0.0764,0.5127,0.2605\}$. Após calcular os pesos para cada fator, os dados dos caminhos de luz são coletados para realizar 
a GRA. O tipo de normalização adotada para cada critério é apresentada na Tabela 3. Finalmente, calculamos o GRC para cada série disponível. Como a opção nominal-melhor não é utilizada, o GRC é dado por

$$
G R C_{i}=\frac{1}{\sum_{p=1}^{4} w_{p}\left|x^{*}(p)-1\right|+1}
$$

Tabela 2. Comparação pareada dos critérios.

\begin{tabular}{|c|c|c|c|c|c|c|c|}
\hline & $(\alpha)$ & $(\beta)$ & $(\theta)$ & $(\gamma)$ & $\begin{array}{l}\text { Média } \\
\text { geométrica (a) }\end{array}$ & $\begin{array}{l}\text { Peso normal } \\
\text { globalizado } \\
\text { (c)=(a)/(b) }\end{array}$ & CI \\
\hline$(\alpha)$ & 1 & 3 & $1 / 3$ & $1 / 3$ & 0,7598 & 0,1504 & \\
\hline$(\beta)$ & $1 / 3$ & 1 & $1 / 5$ & $1 / 3$ & 0,3861 & 0,0764 & 0,0642 \\
\hline$(\theta)$ & 3 & 5 & 1 & 3 & 2,5900 & 0,5127 & \\
\hline$(\gamma)$ & 3 & 3 & $1 / 3$ & 1 & 0,3161 & 0,2605 & \\
\hline
\end{tabular}

Tabela 3. Categoria para a normalização dos caminhos de luz

\begin{tabular}{lcc} 
Parâmetro & Tipo de critério & Categoria \\
\hline CoS $(\alpha)$ & Variável & Maior-melhor \\
Holding-time $(\beta)$ & Variável & Menor-melhor \\
Largura de banda $(\theta)$ & Variável & Maior-melhor \\
Saltos $(\gamma)$ & Variável & Maior-melhor \\
\hline
\end{tabular}

\subsection{Análise de complexidade}

A complexidade computacional do algoritmo proposto foi calculada como: $\mathrm{O}$ algoritmo de Dijkstra utilizado para encontrar a menor distância de um nó s para os demais nós do grafo possui complexidade $O\left(V^{2}\right)$. A complexidade da função utilizada para o cálculo do AHP-GRA é dada como $O(N * K)$, onde $N$ é o número de requisições utilizadas para comparação e $K$ o número de parâmetros para comparação (constante). Logo, consideramos uma complexidade de ordem linear assim como a política de locação de espectro First-Fit. Concluímos que a complexidade do algoritmo é dada por $O\left(V^{2}\right)$.

\section{Resultados numéricos}

Foram realizadas simulações para avaliar o desempenho do algoritmo proposto utilizando o simulador de eventos discretos Simpy baseado em Python. Duas abordagens foram comparadas: sem a adoção do AHP-GRA e com a adoção da abordagem proposta. Replicações independentes foram realizadas para gerar intervalo de confiança de $95 \%$. Cinco simulações foram realizadas para cada ponto das curvas presentes nos gráficos plotados, cada uma com 10000 requisições randomicamente geradas. Estas requisições foram divididas em três classes de serviço. A $\operatorname{CoS} 1$ foi considerada a mais prioritária enquanto que a $\operatorname{CoS} 3$ foi a menos prioritária. Além disso, estas requisições chegavam à 
rede seguindo um processo Poisson e os holding-time destas requisições eram exponencialmente distribuídos com média 2. As demandas possuíam largura de banda de 10Gbps, 20Gbps, 40Gbps e 80Gbps em igual proporção. A topologia de rede NSFNET apresentada na Figura 3 com 14 nós e 21 arestas foi adotada para realização das simulações. Além disso, é possível visualizar a distância adotada para cada uma das arestas presentes (em km).

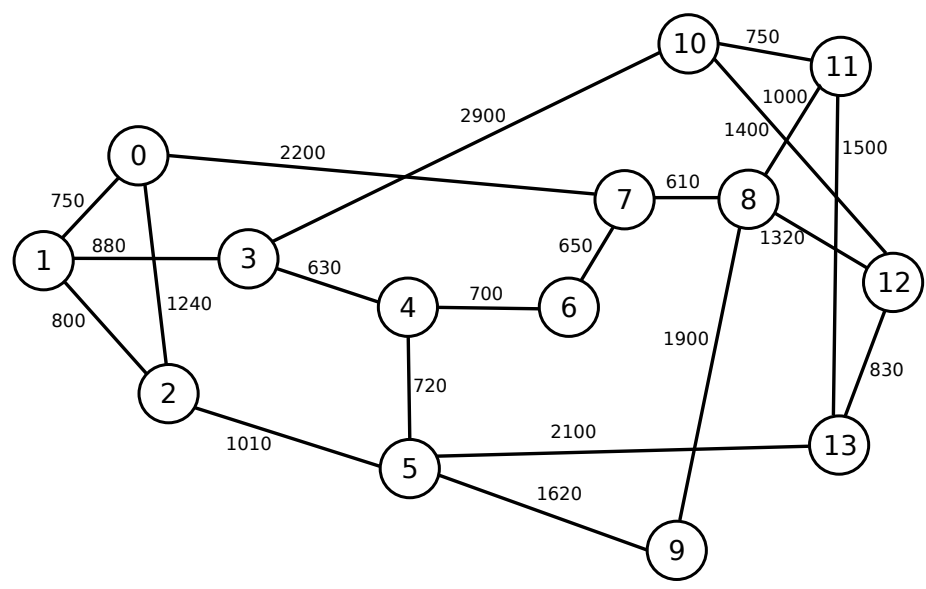

Figura 3. Topologia de rede NSFNET.

Cada enlace da topologia de rede adotada era composto de 300 slots de frequência com 12,5GHz de largura cada. Foram adotados 2 slots de frequência como banda de guarda entre requisições. Os formatos de modulação adotados e seus alcances transparentes (em km) foram BPSK (4000); QPSK (2000); 8-QAM (1000); 16-QAM (500). A política de alocação de espectro adotada nas simulações foi a First-Fit. Foram consideradas as seguintes métricas para estudo: probabilidade de bloqueio (ou seja, a quantidade de requisições bloqueadas em relação ao número total de requisições solicitadas), probabilidade de bloqueio por classe de serviço (ou seja, a quantidade de requisições bloqueadas para cada classe de serviço em relação ao total de requisições solicitadas) e a largura de banda média (ou seja, a quantidade média de largura de banda aceita pela rede).

A Figura 4 apresenta os resultados obtidos para a probabilidade de bloqueio gerados para a classe CoS 1 através de 10 replicações independentes com a carga da rede a 1000 Erlangs. A operação da rede sem a proposta do AHP-GRA apresenta valores altos de probabilidade de bloqueio pra requisições da $\operatorname{CoS} 1$ ao contrário de quando o algoritmo proposto é adotado. Além disso, o conjunto de dados do algoritmo proposto apresenta menos variabilidade que a amostra sem a utilização do algoritmo. O conjunto de dados gerados pelo algoritmo apresenta uma assimetria negativa enquanto que a outra amostra apresenta assimetria positiva.

Como a média das duas amostras de dados na Figura 4 não se sobrepõe, podemos inferir que o algoritmo proposto apresenta baixa probabilidade de bloqueio. Para confirmar essa inferência, foi realizado teste de hipótese através do teste T de Student com as seguintes alternativas:

$$
\begin{array}{ll}
H_{0}: & \mu_{1}=\mu_{2} \\
H_{A} & : \quad \mu_{1} \leq \mu_{2}
\end{array}
$$




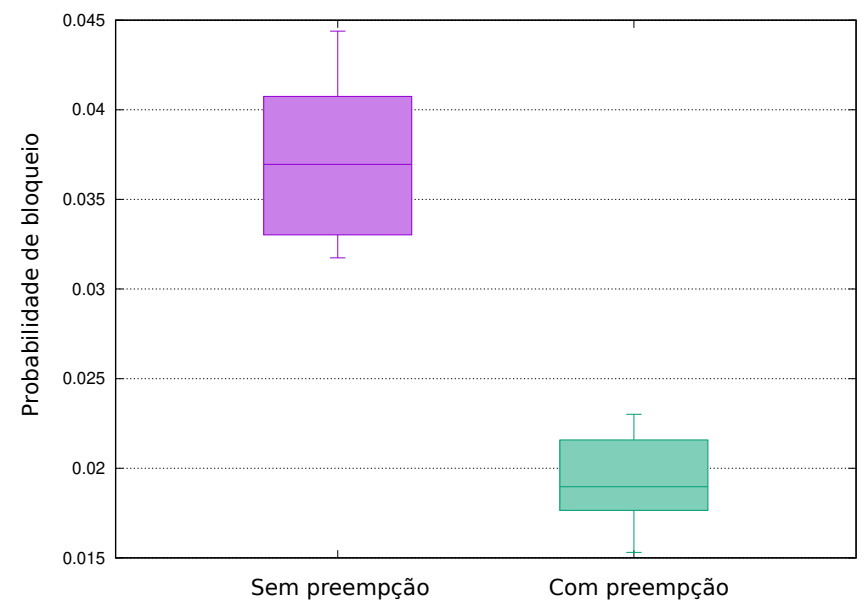

Figura 4. Probabilidade de bloqueio com carga na rede de 1000 Erlangs para a CoS 1.

A Tabela 4 apresenta os resultados utilizados para plotar a Figura 4. Primeiro é avaliado se os dois conjuntos de dados seguem uma distribuição normal usando o teste de Shapiro-Wilk. Como o p-valor para o primeiro e segundo conjunto de dados são 0.613 e 0.662 não rejeitamos a hipótese nula de que os dados seguem uma distribuição normal. Em seguida, é avaliado se os dois conjuntos de dados tem variâncias iguais através do teste de Bartlett. Como o p-valor obtido é 0.199, não rejeitamos a hipótese nula de que os dados possuem variâncias iguais. Finalmente, o p-valor obtido para o teste T de Student é 0,000 , então a hipótese nula de que os conjuntos de dados possuem medias iguais é rejeitada. Desta forma, podemos aceitar que a estratégia proposta é capaz de reduzir a probabilidade de bloqueio das requisições pertencentes a Cos 1.

Tabela 4. Análise das amostras de dados da probabilidade de bloqueio com carga na rede de 1000 Erlangs.

\begin{tabular}{|l|c|c|}
\hline Amostra & Sem preempção & Com preempção \\
\hline $\mathrm{n} 1$ & 0.0407 & 0.0164 \\
\hline $\mathrm{n} 2$ & 0.0365 & 0.0185 \\
\hline $\mathrm{n} 3$ & 0.0411 & 0.0176 \\
\hline $\mathrm{n} 4$ & 0.0444 & 0.0230 \\
\hline $\mathrm{n} 5$ & 0.0317 & 0.0214 \\
\hline $\mathrm{n} 6$ & 0.0330 & 0.216 \\
\hline $\mathrm{n} 7$ & 0.0330 & 0.0153 \\
\hline $\mathrm{n} 8$ & 0.0372 & 0.0179 \\
\hline $\mathrm{n} 9$ & 0.0367 & 0.0218 \\
\hline $\mathrm{n} 10$ & 0.0374 & 0.0195 \\
\hline \multirow{2}{*}{ Teste de Shapiro-Wilk } & $\begin{array}{l}\text { Statistic }=0.945 \\
\text { p-value }=0.613\end{array}$ & $\begin{array}{l}\text { Statistic }=0.949 \\
\text { p-value }=0.662\end{array}$ \\
\hline Teste de Bartlett & Statistic $=1.648$ p-value $=0.199$ \\
\hline Teste T de Student & \multicolumn{2}{|c|}{ Statistic $=11.835$ p-value $=0.000$} \\
\hline
\end{tabular}

A Figura 5 apresenta os resultados obtidos para a métricas probabilidade de bloqueio em estudo. Esta Figura apresenta a probabilidade de bloqueio geral, isto é, para 
todas as CoS. A estratégia proposta foi capaz de reduzir a probabilidade de bloqueio geral da rede. Este resultado está associado com a escolha do caminho de luz que possui mais saltos e maior largura de banda.

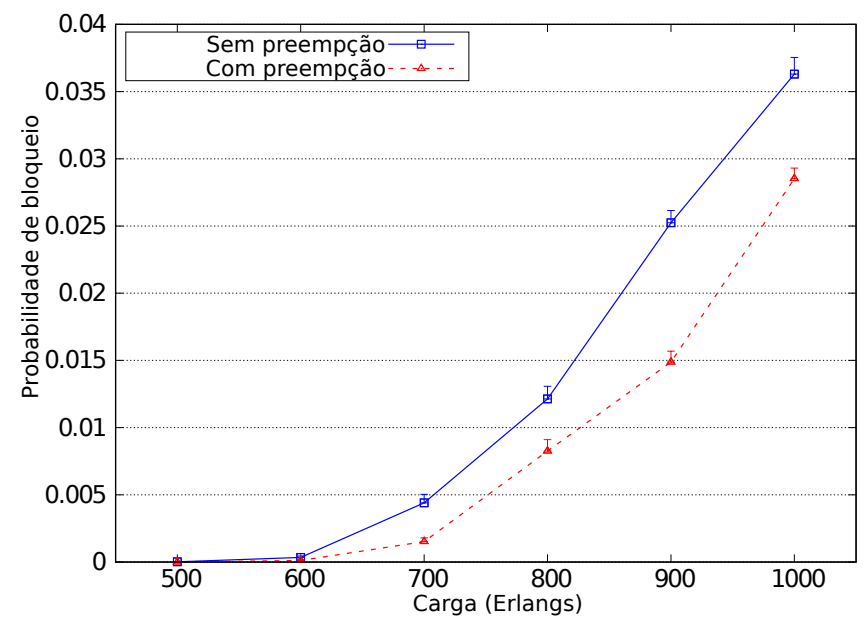

Figura 5. Probabilidade de bloqueio.

Na Figura 6 e Figura 7 onde são apresentados os resultados obtidos para a probabilidade de bloqueio entre as CoSs, vemos mais especificamente na Figura 6 que o uso do AHP-GRA foi mais eficiente na redução de bloqueio das classes de serviços pertencentes a $\operatorname{CoS} 1$. A estratégia proposta foi capaz de reduzir a probabilidade de bloqueio da CoS 1 para cargas acima de 600 Erlangs. Tais resultados confirmam que as escolhas dos parâmetros adotados beneficiam as requisições da $\operatorname{CoS} 1$. Além disso, a Figura 7 apresenta a probabilidade de bloqueio considerando cada $\mathrm{CoS}$ usada nas simulações. Como esperado, $\mathrm{CoS} 3$ foi a que sofreu a maior probabilidade de bloqueio uma vez que ela foi considerada a menos prioritária. A CoS 2 apresentou resultados intermediários, devido a sua importância média.

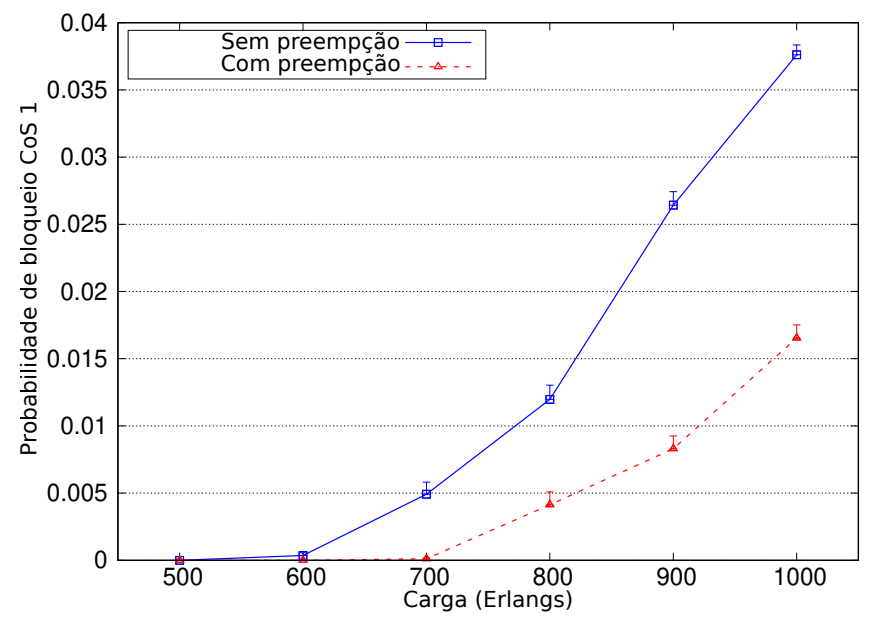

Figura 6. Probabilidade de bloqueio CoS 1.

Na Figura 8 temos a quantidade média de largura de banda aceita pela rede nos dois cenários adotados. O uso do AHP-GRA aumentou a quantidade de largura de banda aceita pela rede. Este resultado pode estar relacionado com a adoção dos parâmetros no AHP-GRA, os quais dão preferência para requisições com maior largura de banda. 


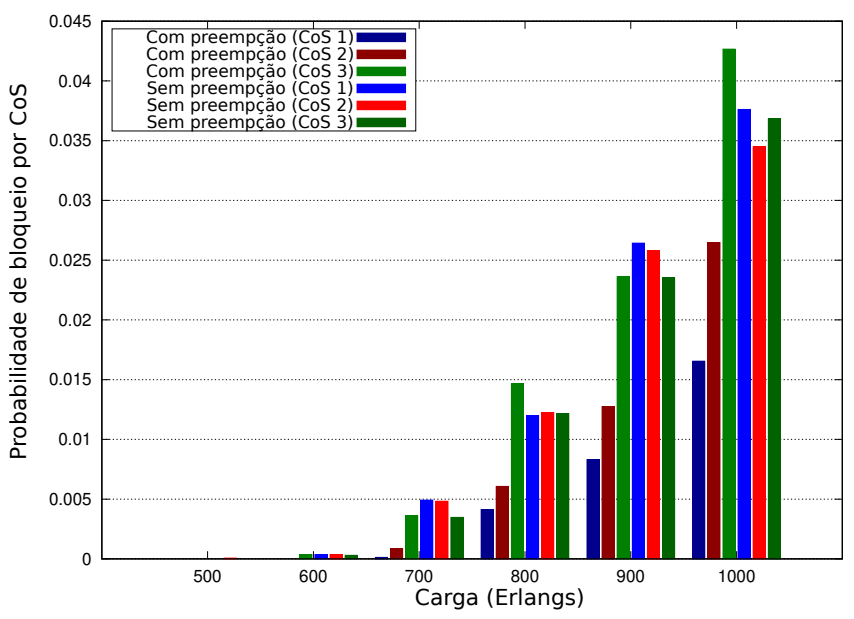

Figura 7. Probabilidade de bloqueio para cada CoS.

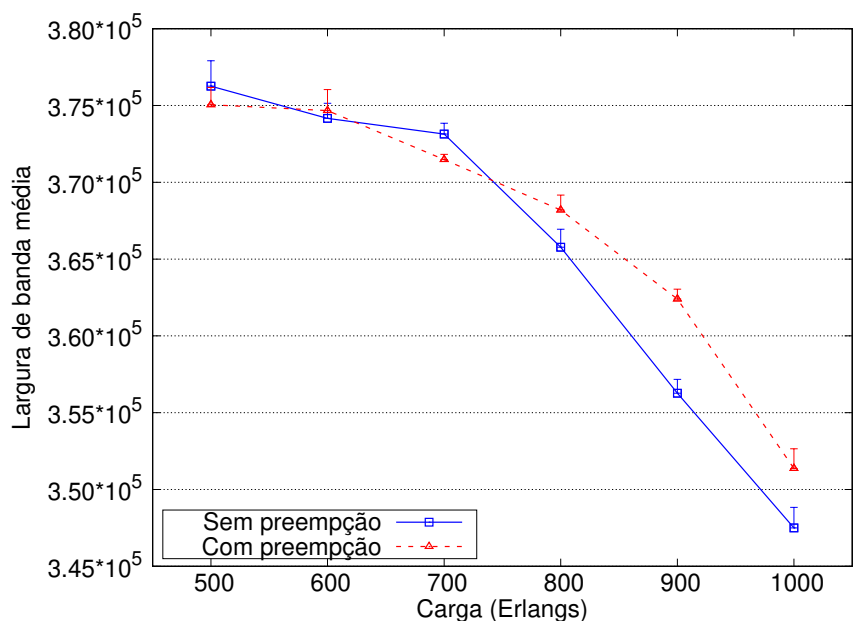

Figura 8. Largura de banda média aceita pela rede.

\section{Análise de sensibilidade}

Foi realizada uma análise de sensibilidade para avaliar como a mudança dos pesos atribuídos aos critérios adotados no AHP interferem nas alternativas que possuem mais importância para a tomada de decisão. Avaliamos se a atribuição de pesos aos fatores largura de banda afeta os pesos já atribuídos aos outros fatores devido ao seu maior peso no vetor prioritário. A análise de sensibilidade é realizada através mudanças de percentagens do peso do critério de interesse [Kabak and Dagdeviren 2017]. O peso do critério desejado e dos demais critérios presentes na análise de sensibilidade são calculados como:

$$
\begin{gathered}
w_{i}=w_{i 0}+w_{i 0} * M P \\
w_{j}=\left(1-w_{i}\right) * \frac{w_{j 0}}{\left(1-w_{i 0}\right)}
\end{gathered}
$$

onde $w_{i}$ é o peso do fator levado em consideração na análise de sensibilidade e $w_{j}$ é o peso atribuído aos demais critérios sobre análise. Os níveis adotados para a análise de sensibilidade foram: $-0,1 ;-0,05 ; 0 ; 0,05 ; 0,1$. A Tabela 5 apresenta os resultados obtidos para cada critério na análise. 
Tabela 5. Pesos atribuídos para a análise de sensibilidade.

\begin{tabular}{lcccc} 
MP & CoS & Holding-time & Largura de banda & Saltos \\
\hline$-0,10$ & 0,1504 & 0,0845 & 0,4614 & 0,2879 \\
$-0,05$ & 0,1583 & 0,0804 & 0,4870 & 0,2742 \\
0,00 & 0,1504 & 0,0764 & 0,5127 & 0,2605 \\
0,05 & 0,1425 & 0,0724 & 0,5383 & 0,2468 \\
0,10 & 0,1346 & 0,0683 & 0,5639 & 0,2330 \\
\hline
\end{tabular}

Com o objetivo de avaliar como a adoção dos pesos para os critérios de decisão no AHP impactam nas variáveis estudadas, realizamos simulações onde os pesos dos critérios possuíam o mesmo valor, ou seja, foi atribuído o valor 1 em todas as comparações pareadas. Tais valores podem ser observados na Tabela 6 .

Tabela 6. Comparação pareada dos critérios.

\begin{tabular}{ccccclll} 
& $(\alpha)$ & $(\beta)$ & $(\theta)$ & $(\gamma)$ & $\begin{array}{l}\text { Média } \\
\text { geométrica (a) }\end{array}$ & $\begin{array}{l}\text { Peso normal } \\
\text { globalizado } \\
(\mathrm{c})=(\mathrm{a}) /(\mathrm{b})\end{array}$ & $\mathrm{CI}$ \\
\hline$(\alpha)$ & 1 & 1 & 1 & 1 & 1 & 0.25 & 0 \\
$(\beta)$ & 1 & 1 & 1 & 1 & 1 & 0.25 & 0.25 \\
$(\theta)$ & 1 & 1 & 1 & 1 & 1 & 0.25 & \\
$(\gamma)$ & 1 & 1 & 1 & 1 & 1 &
\end{tabular}

Os resultados obtidos para a probabilidade de bloqueio geral e da $\operatorname{CoS} 1$ são apresentados na Figura 9 e Figura 10. Os resultados são semelhantes aos encontrados nos resultados anteriores. A abordagem proposta foi capaz de reduzir a probabilidade de bloqueio geral da rede, e manter a redução da probabilidade de bloqueio da $\operatorname{CoS} 1$. Na Figura 11 é notado que a proporcionalidade entre as $\mathrm{CoS}$ é mantida. Este fato é explicado pela preferência adotada no AHP, desde que as requisições de baixa prioridade são preferidas e a escolha do mesmo valor para os parâmetros no AHP não causam interferência neste resultado.

No entanto, adoção de valores iguais para os critérios de decisão no AHP causaram impacto na largura de banda média aceita pela rede como observado na Figura 12. Desta forma, é notado que para aumentar a quantidade de banda que a rede pode receber é importante adicionar um maior peso para o critério de decisão largura de banda como os obtidos no vetor de prioridade nos resultados anteriores.

\section{Conclusão}

Este trabalho apresentou uma proposta para escolher entre bloquear uma requisição que acaba de chegar à rede e não encontra largura de banda suficiente ou realizar a preempção de um caminho de luz já provisionado para aceitar tal requisição. Os resultados obtidos mostram que a estratégia proposta foi capaz de reduzir a probabilidade de bloqueio geral da rede e também das requisições pertencentes à $\operatorname{CoS} 1$. Além disso, foi identificado que atribuir um peso maior para o critério de decisão largura de banda aumenta a quantidade 


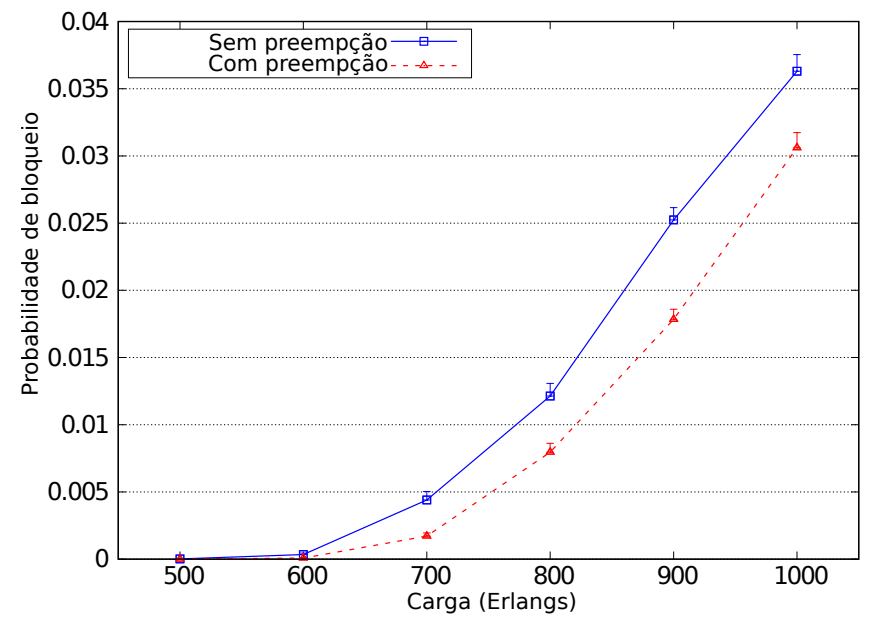

Figura 9. Probabilidade de bloqueio.

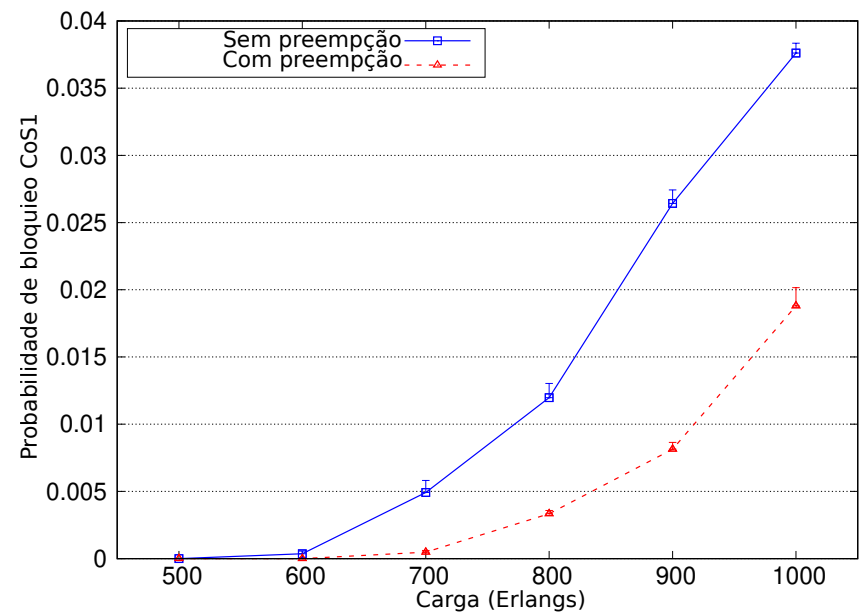

Figura 10. Probabilidade de bloqueio para $\operatorname{CoS} 1$.

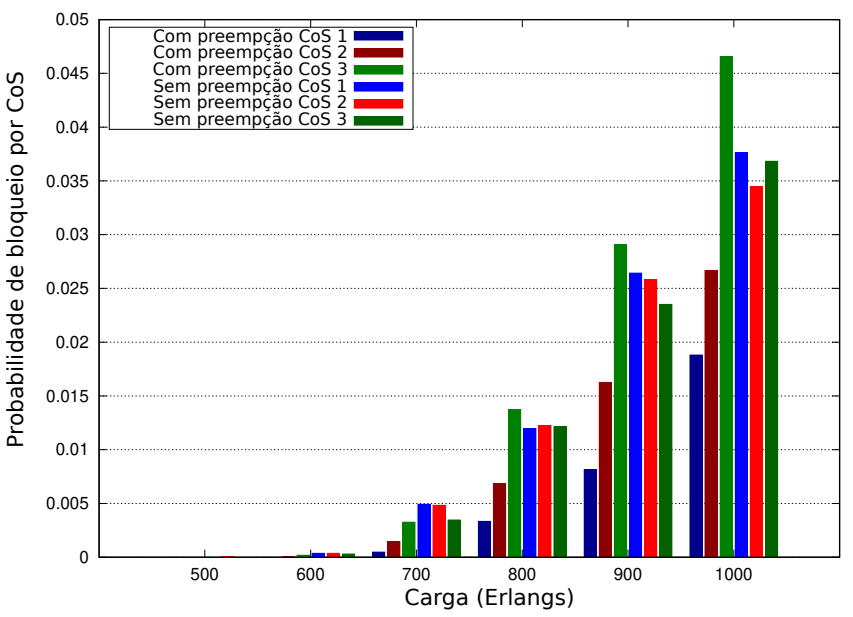

Figura 11. Probabilidade de bloqueio para cada CoS.

de largura de banda aceita pela rede. Com estes resultados, novos estudos serão realizados para entender como as escolhas dos caminhos e a fragmentação presente nas redes EONs podem interferir nos resultados obtidos. 


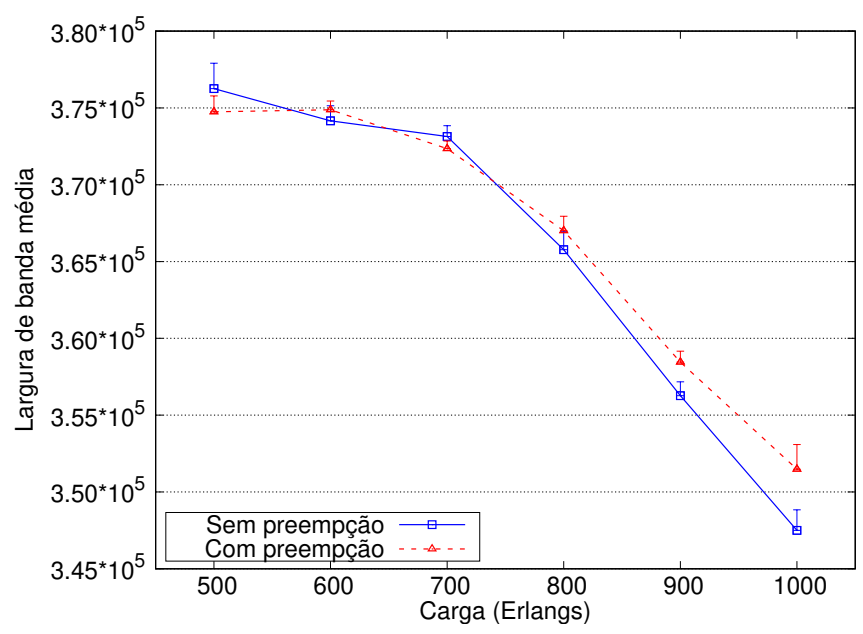

Figura 12. Banda média recebida.

\section{Referências}

Fawaz, W., Chen, K., Nakad, Z., and Abou-Rjeily, C. (2008). A simple quality-of-servicebased connection setup management approach for optical networks. In 2008 IEEE International Conference on Communications, pages 5349-5353.

Fawaz, W., Daheb, B., Audouin, O., Du-Pond, M., and Pujolle, G. (2004). Service level agreement and provisioning in optical networks. IEEE Communications Magazine, 42(1):36-43.

Kabak, M. and Dagdeviren, M. (2017). A hybrid approach based on ANP and grey relational analysis for machine selection. Tehnicki Vjesnik, 24:109-118.

Liu, Y., Zhou, X., Ren, S., Yang, L., and Ci, S. (2012). Peer selection in mobile P2P networks based on AHP and GRA. In 2012 18th IEEE International Conference on Networks (ICON), pages 179-184.

Lu, W., Zhu, Z., and Mukherjee, B. (2015). On hybrid IR and AR service provisioning in elastic optical networks. Journal of Lightwave Technology, 33(22):4659-4670.

Q., S. and A., J. (2005). A network selection mechanism for next generation networks. In IEEE International Conference on Communications, 2005. ICC 2005. 2005, volume 2, pages 1418-1422 Vol. 2.

Saaty, T. L. (1990). How to make a decision: The analytic hierarchy process. European Journal of Operational Research, 48(1):9 - 26. Desicion making by the analytic hierarchy process: Theory and applications.

Sivasankaran, A., Razo, M., Tacca, M., and Fumagalli, A. (2012). Preemption based lightpath restoration. In 2012 IEEE International Conference on Communications (ICC), pages 6252-6256.

Szymanski, A., Lason, A., Rzasa, J., and Jajszczyk, A. (2007). Grade-of-service-based routing in optical networks [quality-of-service-based routing algorithms for heterogeneous networks]. IEEE Communications Magazine, 45(2):82-87. 